\title{
乌ु \\ Performance of solenoids versus quadrupoles in focusing and energy selection of laser accelerated protons
}

\author{
Ingo Hofmann* \\ Gesellschaft für Schwerionenforschung (GSI), Planckstraße 1, 64291 Darmstadt, Germany
}

(Received 29 January 2013; published 3 April 2013)

\begin{abstract}
Using laser accelerated protons or ions for various applications-for example in particle therapy or short-pulse radiographic diagnostics_requires an effective method of focusing and energy selection. We derive an analytical scaling for the performance of a solenoid compared with a doublet/triplet as function of the energy, which is confirmed by TRACEWIN simulations. Generally speaking, the two approaches are equivalent in focusing capability, if parameters are such that the solenoid length approximately equals its diameter. The scaling also shows that this is usually not the case above a few $\mathrm{MeV}$; consequently, a solenoid needs to be pulsed or superconducting, whereas the quadrupoles can remain conventional. It is also important that the transmission of the triplet is found only $25 \%$ lower than that of the equivalent solenoid. Both systems are equally suitable for energy selection based on their chromatic effect as is shown using an initial distribution following the RPA simulation model by Yan et al. [Phys. Rev. Lett. 103, 135001 (2009].
\end{abstract}

DOI: 10.1103/PhysRevSTAB.16.041302

PACS numbers: 41.75.Jv, 87.56.J-

\section{INTRODUCTION}

Laser acceleration of protons or ions requires ultrahigh laser intensities focused on thin target foils. This has been demonstrated in numerous experiments (see, for instance, Refs. [1-4]). Applications of this novel acceleration method have been suggested, for example in terms of a new proton source for radiation therapy [5-7] as an alternative to more conventional accelerator technologies, like protons or ions from cyclotrons or synchrotrons. Other potential applications might be proton radiography, neutron imaging, or isotope production. Energies up to $170 \mathrm{MeV}$ for deuterons have recently been observed at the TRIDENT laser and explained as a "break-out afterburner" mechanism [8], with possible applications as very short-pulse neutron source.

All of these applications have to cope with the characteristics of laser accelerated protons or ions: a large energy spread as well as angular spread, sub-ns time scales, significant shot-to-shot fluctuations, and-for practical applications-relatively low repetition rates compared with conventional accelerators. This requires specific methods to suitably manipulate laser accelerated protons in space and time and match them to the need of an experiment or application. The LIGHT project at GSIusing the PHELIX laser facility-is an experimental effort

\footnotetext{
*Also at Helmholtz-Institut Jena, Helmholtzweg 4, 07743 Jena, Germany.

i.hofmann@gsi.de

Published by the American Physical Society under the terms of the Creative Commons Attribution 3.0 License. Further distribution of this work must maintain attribution to the author(s) and the published article's title, journal citation, and DOI.
}

to explore these issues with regard to possible future applications in areas like proton radiography, warm dense matter, material science, biophysics, or particle therapy. Preliminary results on the LIGHT project are discussed in Ref. [9]. Our findings are of direct relevance for the LIGHT project whenever the proton energy rises from above a few $\mathrm{MeV}$ into the range of several tens or even a hundred $\mathrm{MeV}$.

In a preceding study we have shown that a single solenoid magnet can be used very effectively to combine angular focusing (collection) with energy selection due to the lens chromatic effect. Relevant background material motivating the present study is found in Ref. [10]. The key issue here is that this is specifically relevant to laser acceleration, where a large inherent energy spread appears simultaneously with a large angular spread. This subject is extended here to a comparative evaluation of quadrupole focusing (doublet or triplet) and solenoid focusing. As in the solenoid case, we use the dependence of focal length on energy and employ a radially confining aperture to select a suitable energy window. The main difference of quadrupoles versus a solenoid is their first order focusing property (solenoids focus in second order) and the asymmetry in focusing (astigmatism) as well as different chromatic effects between the horizontal and vertical planes. On the other hand, alternating gradient focusing with quadrupoles is known to be more efficient at increasing energy and helps to avoid pulsed magnet or superconducting technology.

It should be mentioned here that an alternative to energy selection by the chromatic effect is the more conventional energy selection using the dispersive properties of dipole magnets. All therapy oriented studies on laser acceleration carried out in the course of the past years have used such a system with a collimating aperture followed by a 
dispersive dipole magnet [11-14]. Dispersive energy selection is certainly an option, but schemes without transverse focusing may result in significant efficiency loss. Chromatic energy selection, instead, combines the advantages of focusing with energy selection in a single device. However, for effective selection it is required that the beam size by chromaticity dominates over the size by the intrinsic emittance, which is not the case in many situationsbut certainly for laser accelerated particles.

An interesting alternative is the microlens, which focuses by means of an electron plasma initialized by a second short-pulse laser beam and collapsing inside a cylinder [15]. Energy selection is enabled by using time of flight and triggering the second laser beam accordingly. For therapy applications, however, where energy selection failure cannot be tolerated, the fully controllable powering of conventional magnets for energy selection seems essential.

In Sec. II we compare solenoidal with doublet/triplet focusing. Their chromatic focusing properties are studied in Sec. III. In Sec. IV results are applied to energy selection by an aperture for both systems using an input distribution from the simulation of a radiation pressure acceleration model. In Sec. V we draw conclusions.

\section{COMPARISON OF SOLENOIDAL AND QUADRUPOLAR FOCUSING}

Solenoids are frequently applied in injectors for focusing of particles with relatively low energy and large divergence. Likewise, focusing of laser accelerated protons with energies of the order of $10 \mathrm{MeV}$ was demonstrated using a pulsed solenoid $[9,16]$. Other laser proton experiments in a comparable energy range have successfully employed small aperture, high-gradient permanent magnet quadrupoles [17]. Preference of quadrupoles over solenoids depends on the individual application, but certainly energy and the question of room temperature, nonpulsed quadrupoles versus pulsed or superconducting solenoids matter.

A useful guidance to decide on the basis of required field strengths can be obtained from a scaling expression using the thin lens approximations for the focal length $f_{s}$ of a solenoid and $F_{d}$ of a quadrupole doublet as suggested in Ref. [18]. With $B$ the field strength (for the quadrupoles defined at the poles), and assuming that both focal lengths are defined from the respective centers, we have for the solenoid of length $L$,

$$
1 / f_{s} \approx\left(\frac{q}{2 m c \beta \gamma}\right)^{2} B^{2} L
$$

likewise for the doublet

$$
1 / F_{d} \approx\left(\frac{q B}{m c \beta \gamma a}\right)^{2} l^{2} s
$$

where $l$ is the individual quadrupole length, $s$ the separation of quadrupoles (from center to center), and $a$ the maximum beam radius (pole radius). Note that the focal strength of a doublet increases with the separation of its components - of course on the expense of decreasing acceptance. Comparing a solenoid with a doublet of the same overall length $L$ and equal field $B$, we readily obtain from Eqs. (1) and (2) the ratio $T_{d}$ of focusing strengths (here defined as inverse focal lengths) in terms of only geometrical quantities:

$$
T_{d} \equiv \frac{1 / F_{d}}{1 / f_{s}}=\frac{4 s l^{2}}{a^{2} L} .
$$

Equation (3) indicates that the focusing strength of a doublet is superior to that of a solenoid, if $a$ is sufficiently small relative to the length. As an example, consider a doublet with a gap between magnets equal to their length, in which case we have $T_{d}=(2 / 3)^{3}(L / a)^{2}$ and the transition condition $T_{d}>1$ occurs for $L / a>(3 / 2)^{3 / 2} \approx 1.8$. Hence, the focusing equivalence with this doublet is given, if the solenoid length approximately equals the aperture diameter. In physical terms this implies that the solenoid fringe field region length is comparable with the core length. This, in turn, reflects the fact that the solenoid focusing is second order in the sense that the fringe field region first provides a beam rotation, which is translated into focusing by means of the core longitudinal field. In quadrupoles the azimuthal field directly leads to focusing, instead.

For $T_{d}$ deviating from unity systems with equal focal lengths can still be achieved by adjusting $B_{s}$ according to Eq. (1). This results in an effective field for the solenoid,

$$
B_{s}^{*}=T_{d}^{1 / 2} B_{d},
$$

which may lead to the requirement of superconducting or pulsed power technologies for the solenoid.

We can apply this to the collection of laser particles, if we assume the focal spot is at the source and the beam is to be made parallel by the lens. In the interest of smoother focusing, we find it preferable to use a triplet rather than a doublet as a reference case. In Fig. 1 we show-as example-a triplet with $L=0.334 \mathrm{~m}, l=0.06 \mathrm{~m}$ and gradients of $30 \mathrm{~T} / \mathrm{m},-30 \mathrm{~T} / \mathrm{m}$, and $15 \mathrm{~T} / \mathrm{m}$. The calculation of matched beam optics is obtained using the envelope option of the TRACEWIN code [19], which is also employed further below for particle tracking.

$2 \mathrm{MeV}$ protons with source divergence of $\pm 125 \mathrm{mrad}$ are made parallel with a triplet focal length (source to triplet center) $F_{t}=0.254 \mathrm{~m}$, maximum envelope $a=48 \mathrm{~mm}$, hence maximum pole-tip field $B_{t}=1.44 \mathrm{~T}$. Note that the distance source to lens is given by $F_{t}-L / 2$, which is $87 \mathrm{~mm}$ in this case. The energy spread is assumed to be zero, which is to avoid the chromatic energy effect at this point. For comparison, we also show a solenoid focusing with the same $L$; in order to achieve the same 

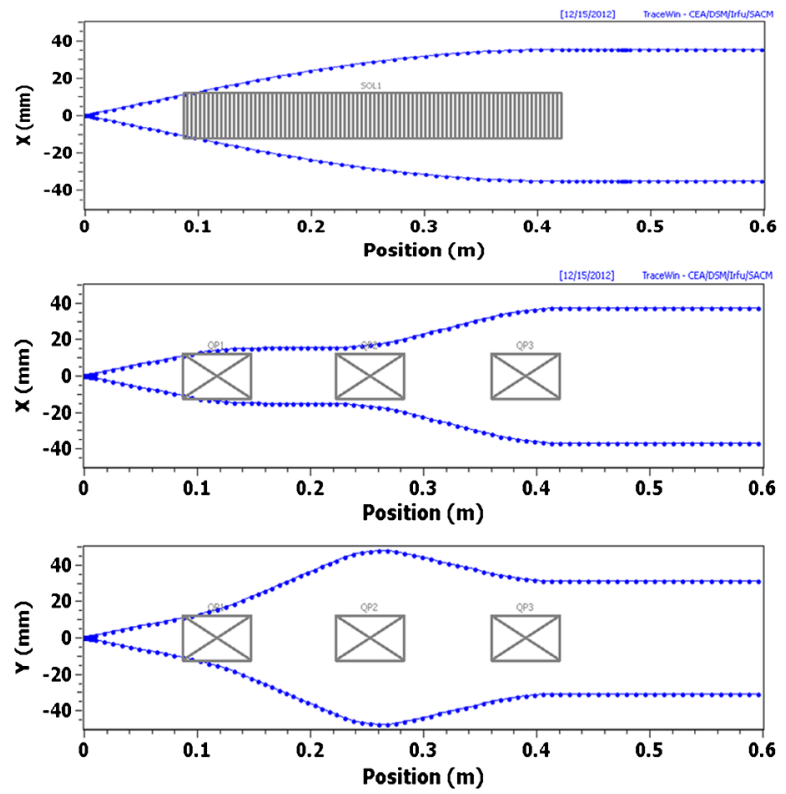

FIG. 1. TRACEWIN envelopes for equivalent solenoid (top) and triplet (center and bottom) solutions at $2 \mathrm{MeV}$.

focal length we require a slightly increased magnetic field $B_{s}=1.53 \mathrm{~T}$. Next we check if our TRACEWIN results still obey the scaling of Eq. (3), although we have replaced the doublet by a triplet. We find good agreement, if we replace $4 s$ by $2 s$. The need for this adjustment can be interpreted as necessary compensation of the (approximate) doubling of the triplet length compared with that of the doublet. This suggests a triplet focusing enhancement factor,

$$
T_{t} \equiv \frac{1 / F_{t}}{1 / f_{s}}=\frac{2 s l^{2}}{a^{2} L}
$$

Assuming that the gap equals the quadrupole length, we have $s=2 l$, which results in $T_{t} \approx 1.12$ in the above example. Applying Eq. (4) we find that the predicted solenoid field for equivalent focal length is $1.52 \mathrm{~T}$, which agrees quite well with the above TRACEWIN result-in spite of the thin lens approximations employed in the derivation of $T_{t}$.

In order to further examine the validity of Eq. (5) for different energies we extend the systems in Fig. 1 to proton energies of $0.2,20$, and $200 \mathrm{MeV}$, again assuming at each energy equal overall lengths $L$ for solenoid and triplet, equal focal lengths for the two systems as well as equal gap and quadrupole lengths in the triplet case. For the initial divergence we assume-somewhat arbitrarily-a divergence scaling $x^{\prime} \propto(\beta \gamma)^{-1 / 2}$ to account for the expected trend of decreasing divergence with energy. The value of $x^{\prime}$ at $2 \mathrm{MeV}$ is chosen as before. We first use TRACEWIN and search again for matched solutions requiring a parallel output beam for vanishing energy spread.

In Table I we summarize all relevant parameters including the resulting quadrupole pole-tip fields and solenoid strengths. The focal length $F$ is again defined from source
TABLE I. Comparison simulation-theory for equivalent solenoid and triplet focusing properties; lengths in $\mathrm{cm}$ and magnetic fields in $\mathrm{T}$ ( $x^{\prime}$ in mrad).

\begin{tabular}{lrcccccccc}
\hline \hline $\mathrm{E}(\mathrm{MeV})$ & \multicolumn{1}{c}{$\mathrm{x}^{\prime}$} & \multicolumn{1}{c}{$\mathrm{L}$} & $\mathrm{F}$ & 1 & $\mathrm{a}$ & $\mathrm{B}_{t}$ & $\mathrm{~B}_{s}^{\mathrm{TW}}$ & $\mathrm{T}_{t}$ & $\mathrm{~B}_{s}^{*}$ \\
\hline 0.2 & 400 & 25.0 & 22.5 & 2 & 5.3 & 1.60 & 0.59 & 0.046 & 0.34 \\
2 & 125 & 33.4 & 25.4 & 6 & 4.8 & 1.44 & 1.53 & 1.12 & 1.52 \\
20 & 71 & 62.0 & 47.0 & 10 & 4.8 & 1.50 & 2.62 & 2.8 & 2.51 \\
200 & 39 & 108.0 & 79.0 & 20 & 4.5 & 1.35 & 5.10 & 14.6 & 5.16 \\
\hline \hline
\end{tabular}

to center of the respective lens system and found to increase with energy. The theoretically expected triplet enhancement factor $T_{t}$ is calculated from Eq. (5) $(s=2 l)$ by inserting the respective geometrical dimensions. Using Eq. (4), which applies equally to the pole-tip field of the triplet as it does for the doublet, we can thus derive the theoretically expected $\mathrm{B}_{s}{ }^{*}$ and compare it with the actual $\mathrm{B}_{s}^{T W}$ obtained from TRACEWIN matching. As result we find an overall good agreement between $\mathrm{B}_{s}^{T W}$ and $\mathrm{B}_{s}^{*}$, which confirms the theoretically derived triplet focusing enhancement over a solenoid. In Fig. 2 we summarize the main findings from this comparison.

Note that the decrease of divergence $x^{\prime}$ with increasing energy assumed in the above used scaling for $x^{\prime}$ is essential for the quadrupolar efficiency taking over. If, unrealistically, the beam source divergence would increase with energy such that $L \approx 2 a$, the focusing equivalent solenoid could keep the same field strength as the quadrupole poletip field independent of energy. This reflects the purely geometrical nature of Eq. (3).

In summary, this demonstrates that for sub-MeV or few $\mathrm{MeV}$ energies solenoids are a convenient approach, whereas the quadrupole doublet/triplet (or multiplet) has advantages for higher energies as its pole-tip field strengths remain within iron saturation. Equation (5) also suggests that $T_{t} \propto 1 / a^{2}$, hence larger $a$ due to an increased initial divergence shifts the advantage of the doublet/triplet over the solenoid to higher energies - and vice versa.

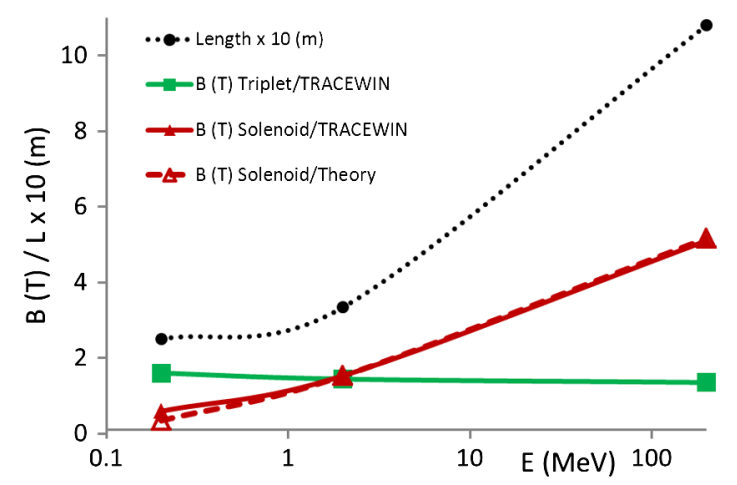

FIG. 2. Comparison of triplet and solenoid systems as a function of energy, where theory focal lengths have been made equal by adjusting the solenoid $\mathrm{B}$ field; $\mathrm{B}_{s}^{*}$ (dashed) from theory and $\mathrm{B}_{s}^{\mathrm{TW}}$ (continuous) from TRACEWIN. 


\section{CHROMATIC PROPERTIES OF SOLENOID AND TRIPLET}

TRACEWIN is used here for particle tracking. Although primarily a linear accelerator design and verification tool, it has a number of features, which make the code suitable for our problem as well. In particular, it (i) is a selfconsistent 3D particle-in-cell code suitable for tracking of up to $10^{7}$ simulation particles, (ii) is capable of energy dependent focusing (chromatic aberrations), (iii) includes field map options for magnetic elements (like solenoids) to model higher order (for example geometric) lens aberrations, (iv) provides standard 6D phase space initial distributions as well as user provided input distributions; as "standard uniform" we choose for this study the option of a uniform distribution in the 4D transverse space as well as uniform within the longitudinal phase plane ellipse, and (v) includes an envelope option for beam optics design.

Space charge options with 2D/3D Poisson solvers exist, but space charge is ignored in this study. In Ref. [10] it is shown for solenoids that space charge is generally weak; in the near-source region, where extremely high proton densities are prevailing, neutralization by the comoving electrons helps.

In the following we use as reference a solenoid and an equivalent triplet, both $104 \mathrm{~cm}$ long and designed to bring $250 \mathrm{MeV}$ protons to a focus at $2.73 \mathrm{~m}$ with the following assumptions: (i) initial maximum divergence angle: \pm 28 mrad; (ii) energy spread: practically monoenergetic; (iii) distance laser target-first magnet: $35 \mathrm{~cm}$; (iv) beam pipe radius: $3.5 \mathrm{~cm}$; (v) aperture radius of solenoids and quadrupoles: $3 \mathrm{~cm}$; (vi) length of solenoid field map: $104 \mathrm{~cm}$; (vii) length of solenoid field region: $80 \mathrm{~cm}$; (viii) averaged solenoid field: $6.27 \mathrm{~T}$; (ix) length of triplet system: $104 \mathrm{~cm}$; (x) length of quadrupoles: $15 \mathrm{~cm}$; and (xi) quadrupole pole-tip fields: 1.5/1.5/1.0 T.

Figure 3 shows density plots from a multiparticle simulation using a low number of simulation particles (only 3000), which helps to visualize single particle rays. The simulation was carried out with the "standard uniform" initial distribution of TRACEWIN. Maximum energy deviations in this example have been chosen as $\pm 5 \times 10^{-4} \mathrm{MeV}$ centered at $250 \mathrm{MeV}$, hence practically monoenergetic. The common waist for $x$ and $y$ for the triplet (stigmatic image) is relevant for optimum energy selection as will be shown in the next section. It is noticed that the defocusing effect of the first quadrupole in $y$ leads to a-in this example-small beam loss at the aperture of the second quadrupole.

In Ref. [10] the energy dependence of the focal length of a solenoid lens was expressed in terms of a chromatic coefficient, which we generalize here to cope with the different focusing in $x$ and $y$ for a triplet:

$$
\alpha_{x, y} \equiv \frac{\delta f_{x, y} / f_{x, y}}{\delta E / E}
$$

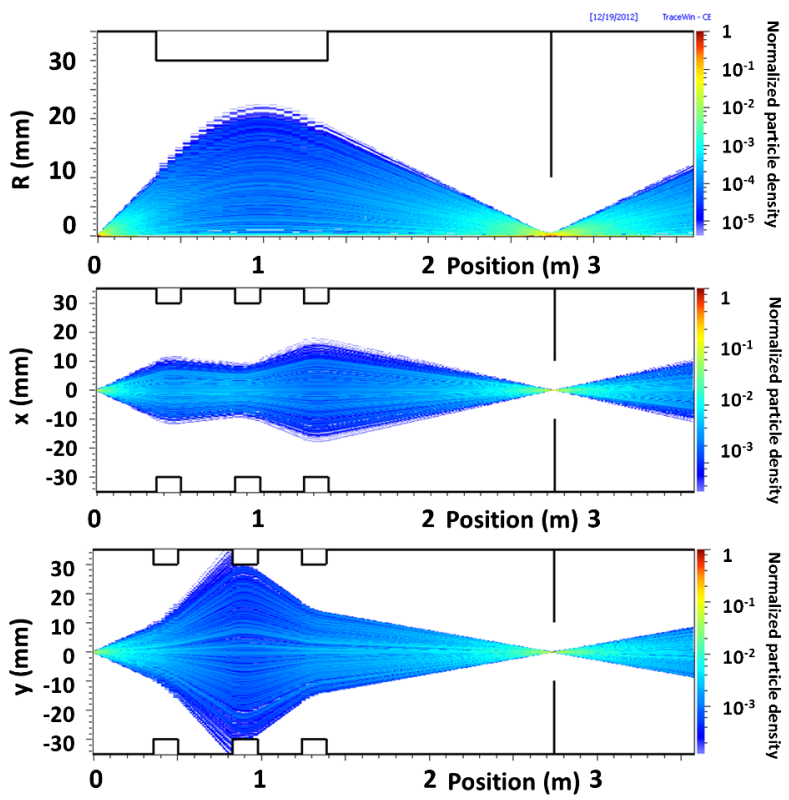

FIG. 3. Density plots for TRACEWIN obtained reference cases for equivalent solenoid (top) and triplet (center: $x$; bottom: $y$ ) focusing.

Here $f_{x, y}$ is the focal length at the reference energy $E$ and $\alpha_{x, y}$ is specific to the geometry of the focusing setup. For the examples of Fig. 3 we find from TRACEWIN simulation for the solenoid $\alpha_{r} \approx 1.9$ and for the triplet $\alpha_{x} \approx 0.9$ as well as $\alpha_{y} \approx 3.8$. The much larger $\alpha_{y}$ is a result of the defocusing in $y$ at the first lens and the thus much larger overall envelope excursions in $y$. The solenoid chromatic coefficient is - not surprisingly - close to the geometrical mean of the two coefficients for the equivalent triplet.

The chromatic effect is strongly correlated with time as higher energy particles travel ahead. The stronger focusing for lower energies leads to an enhanced transverse phase space rotation of protons at the bunch end as compared with the high energy particles at the bunch head. The resulting slip in the transverse phase planes causes an effective transverse emittance increase, which can significantly exceed the initially small production emittance. The effective emittance is obtained by averaging the "slice" (instantaneous) emittances over the full bunch length, hence the full energy spectrum [10].

\section{TRANSMISSION AND ENERGY SELECTION}

As suggested in Ref. [10], the pronounced chromatic focusing effect can be used for an effective energy selection, if the beam is focused into a suitably defined transverse aperture. Only particles with focal spot sufficiently close to the aperture plane are transmitted effectively. As for solenoids this works effectively only if the beam is "chromaticity dominated": at a selection aperture the beam size by chromaticity dominates over the size generated by the intrinsic emittance at any relevant value of the 
energy (i.e. position along the bunch). This is always the case for laser generated ions with their extremely small emittance at any given energy, which is owed to the very small source spot size. It should be mentioned here that the intrinsic emittance should also include emittance increase due to higher order aberrations of the lens, which can be a problem for short solenoid lenses at low energy but is not further considered in this study.

\section{A. RPA generated initial distribution}

For practical considerations it is advantageous to consider an initial distribution in $6 \mathrm{D}$ phase space with a broadened energy distribution according to some laser acceleration model. The radiation pressure acceleration (RPA) mechanism [20-25] has a high potential to reach proton energies of hundreds of $\mathrm{MeV}$. A specific theoretical version of it has been discussed in Ref. [26] and applied to proton therapy conditions in Refs. $[10,27]$ to create a proton energy spectrum extending up to $250 \mathrm{MeV}$. The output of this RPA simulation can be described as spectral yield,

$$
\frac{d N(E, \Omega)}{d E}\left[\mathrm{MeV}^{-1}\right]
$$

which describes the number of particles in an energy interval $d E$ and within a cone angle $\pm \Omega$. The thus defined proton spectrum (for details see Ref. [27]) is plotted in Fig. 4. Its energy distribution is peaked above $200 \mathrm{MeV}-$ determined by the laser intensity - with a relatively broad foot towards lower energies. As input into our TRACEWIN simulations, we take a bi-Gaussian approximation to this simulated energy spectrum shown by the continuous curve in Fig. 4. The 6D initial distribution is taken as a Gaussian random distribution in the variables $t, x, x^{\prime}, y, y^{\prime}$ employing $10^{6}$ simulation particles. For the rms widths in $x^{\prime}, y^{\prime}$ we have chosen $35 \mathrm{mrad}$ - in contrast with the broader tails in

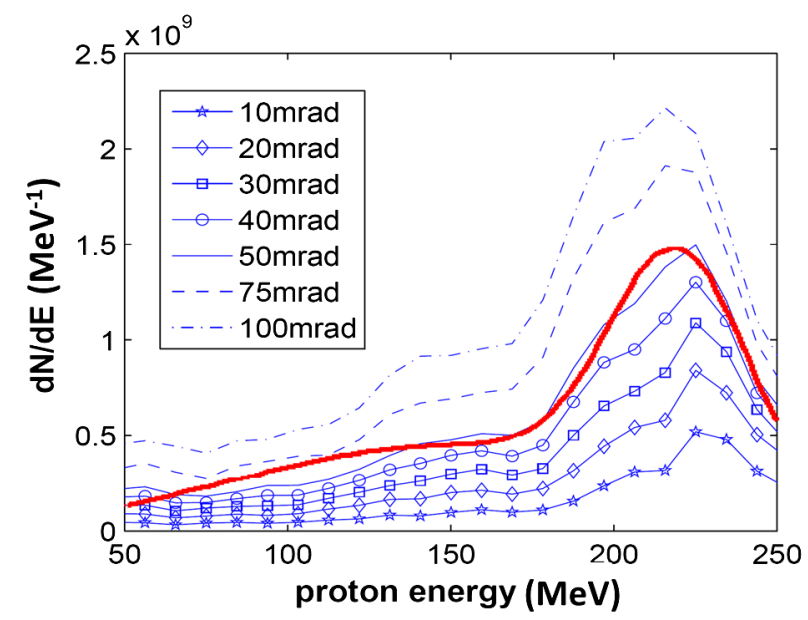

FIG. 4. Simulated spectral yield of protons as a function of energy and for different capture cone angles $\Omega$, with bi-Gaussian fit (continuous line). divergence indicated in Fig. 4, which are probably due to the 2D nature of the RPA simulation. The initial spot radius is in the $\mu \mathrm{m}$ scale, and the pulse duration in the ps scale; their actual values play no role as long as space charge is considered as neutralized initially. We also note that the detailed profile of the energy spectrum is only exemplary and sensitively dependent on details of the code and the modeling, which cannot claim proximity to expected future experimental results. What matters primarily for our purpose of energy selection is the intensity and gradient near the selected energy.

\section{B. Comparative transmission}

For this purpose we reduce the magnet fields in the equivalent solenoid and triplet systems of Fig. 3 for nominal transmission at $220 \mathrm{MeV}$, which is closer to the peak of the energy spectrum. We also require-arbitrarily-a focus at the distance of $2.73 \mathrm{~m}$ from the laser target, where the energy selection aperture is placed. Employing the above defined RPA distribution and a $3 \mathrm{~mm}$ radius aperture, the resulting orbits of a TRACEWIN simulation with 3000 rays are shown in Fig. 5. In Fig. 6 we examine the transverse emittances for the triplet of Fig. 5. The relatively large spread in $x^{\prime}, y^{\prime}$ and energy width together with the energy dependent focusing result in significant emittance growth in $x$ and $y$ within the quadrupoles, accompanied by emittance reductions due to beam loss on the radial aperture. Note that emittances are understood here as averaged
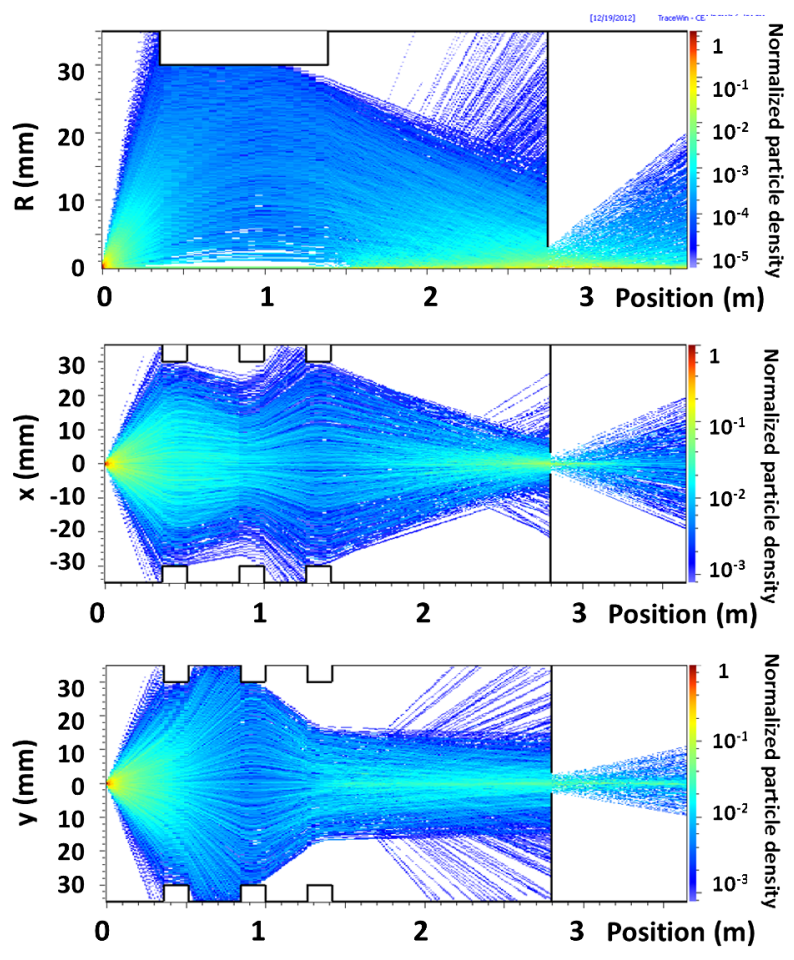

FIG. 5. Density plots for RPA distribution in equivalent solenoid (top) and triplet (center: $x$; bottom: $y$ ) systems adjusted to $220 \mathrm{MeV}$. 


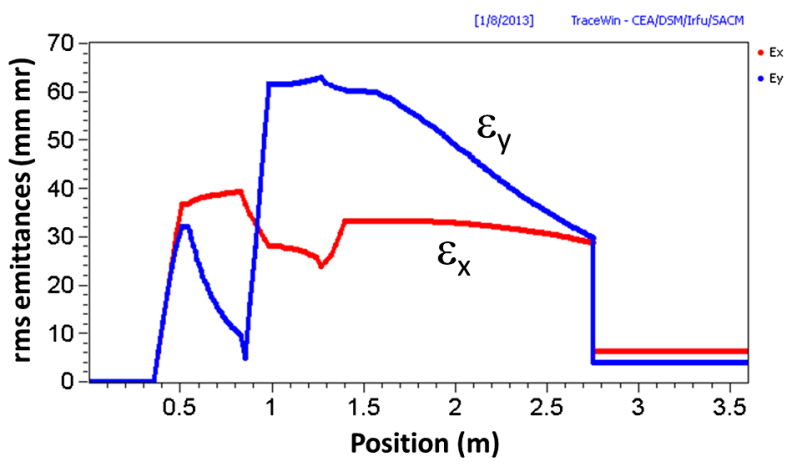

FIG. 6. Transverse (normalized and bunch averaged) rms emittances for triplet case.

over the full bunch length, while the slice emittances at a given position along the expanding bunch remain at their small initial values. Also note that the starting transverse emittances (rms normalized) taken over from the RPA simulations and used here are as small as $0.04 \mathrm{~mm} \mathrm{mrad}$, hence the chromatic effect is huge. The emittance reduction in $y$ within the first quadrupole reflects the beam loss in the defocusing $y$ direction. A comparison of the equivalent solenoid and triplet focusing systems shows that the overall transmission for the solenoid is $47 \%$, and $35 \%$ for the triplet.

The corresponding loss profiles are shown in Fig. 7. The beam loss in $y$ in the first lens of the triplet is to some extent compensated by an enhanced transmission in $x$, which explains why the triplet transmission is only $25 \%$ lower than the solenoid one. A more differentiated insight is gained, if we truncate the divergence of the initial Gaussian distribution in $x^{\prime}, y^{\prime}$ by eliminating all particles beyond a "divergence limit" as shown in Fig. 8. Below about $40 \mathrm{mrad}$ the solenoid accepts all injected particles in the truncated distribution (intensity in it relative to untruncated distribution indicated by the dotted line); at this value the acceptance limit is reached and larger divergence

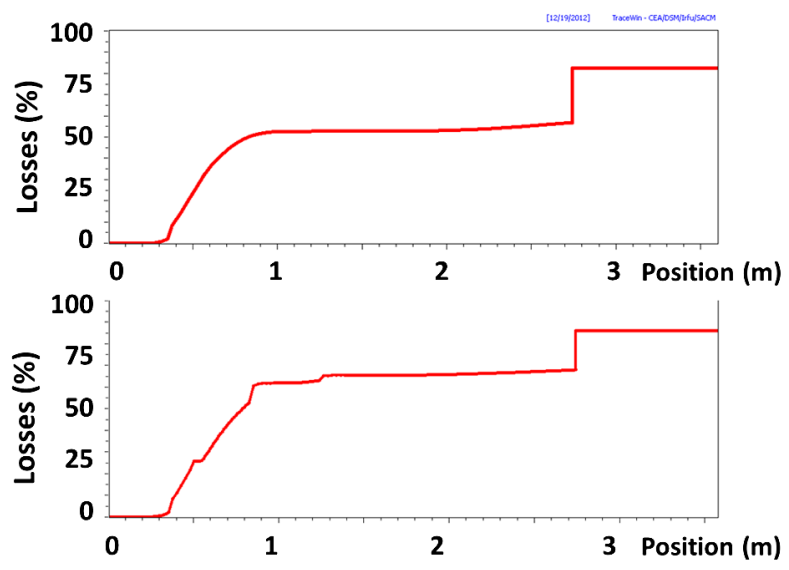

FIG. 7. Loss profiles for RPA distributions in the equivalent solenoid (top) and triplet (bottom).

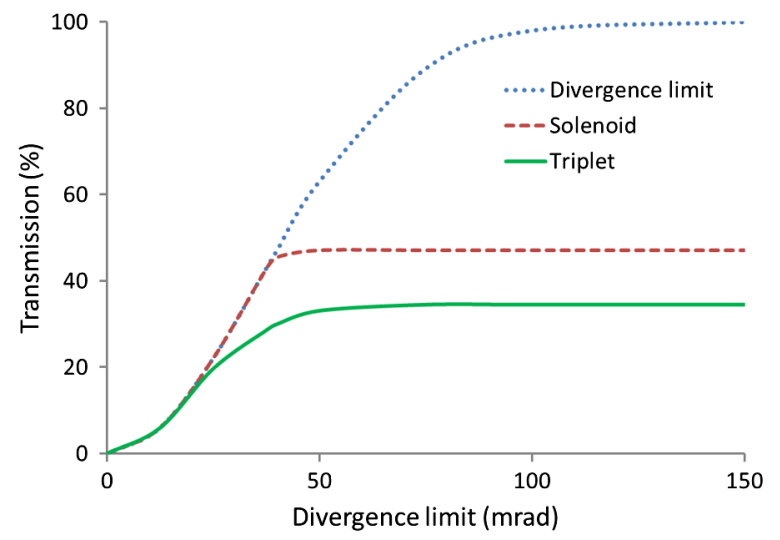

FIG. 8. Transmission of solenoid and triplet as function of an upper cutoff (divergence limit) of the injected particles. The transmission is in $\%$ of the particles in the original untruncated distribution.

particles are lost. The triplet, instead, starts losing particles above $20 \mathrm{mrad}$, but the transition to its acceptance limit is smoother-apparently due to the benefit from the horizontal plane.

\section{Selection of energies}

Following Ref. [10], the radius of a selection aperture is proportional to the product of required energy width and chromatic coefficient $\alpha$,

$$
R_{A}=\alpha \frac{\Delta E}{E} A_{\max },
$$

where $A_{\max }$ is the maximum envelope at the lens. Using $A_{\max } \approx 3 \mathrm{~cm}$ and $\alpha \approx 2$, we expect that an energy width of $\pm 4 \%( \pm 8.8 \mathrm{MeV})$ should be obtainable with an aperture of $2.4 \mathrm{~mm}$ radius. This is approximately confirmed by the energy spectra in Fig. 9, where $R_{A}$ was chosen as $3 \mathrm{~mm}$ for the solenoid and $2.7 \mathrm{~mm}$ for the triplet to reach the same FWHM width. The overall yield in the selected energy windows is $17 \%$ for the solenoid and $13 \%$ for the triplet, which follows approximately the $25 \%$ triplet transmission reduction found in Fig. 8. The "plateaus" in the selected profiles reproduce well the "plateau" of the unselected distribution at $220 \mathrm{MeV}$. The jitter is caused by statistics with the limited number of simulation particles. Note that the triplet plateau is slightly more narrow, instead. For a possible explanation of this slight "imbalance" one needs to note that the $25 \%$ lower transmission of particles in the triplet has eliminated larger angle and energy deviation particles-having more difficulty to pass the aperturehence, the phase space distribution is also modified and not only the intensity.

For the triplet we have also simulated an elliptical selection aperture in $x, y$ with the same area but semiaxes in the ratio 1:4 to match the ratio of chromatic coefficients according to Eq. (8). However, we obtain practically the same energy selection width and profile as well as 

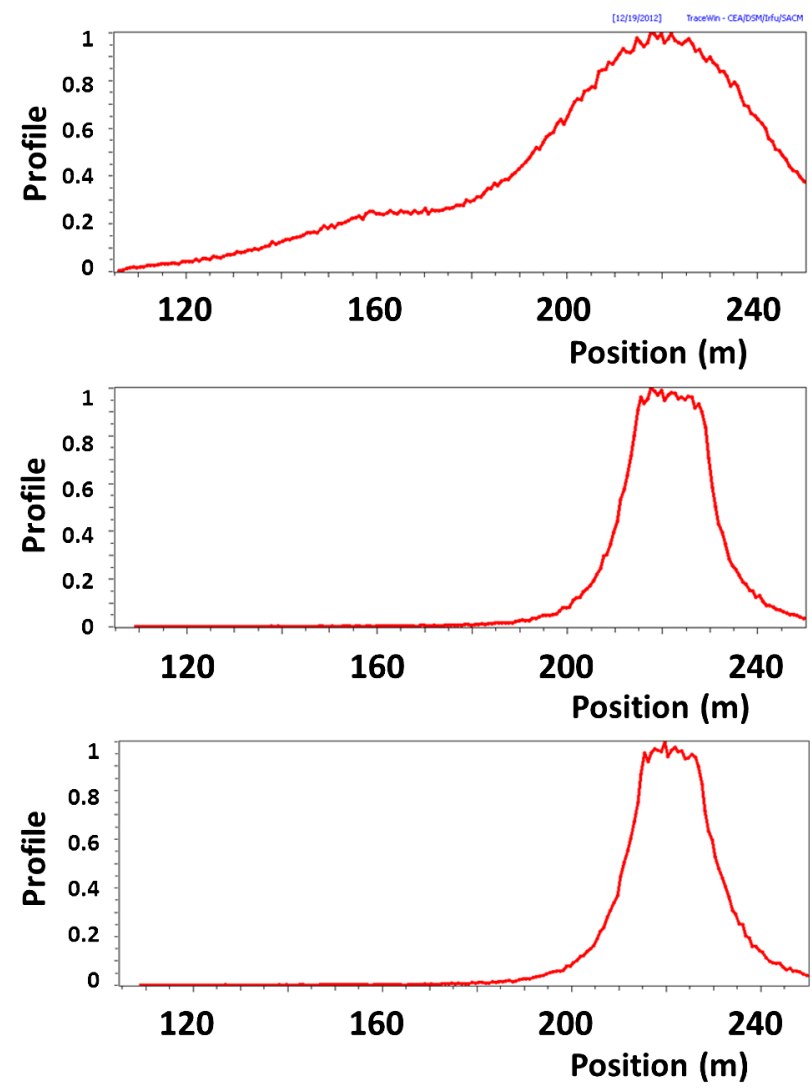

FIG. 9. Selected energy profiles (normalized to unity) for equivalent solenoid $\left(R_{A}=3 \mathrm{~mm}\right)$ and triplet systems $\left(R_{A}=\right.$ $2.7 \mathrm{~mm})$. Top: initial; center: solenoid; bottom: triplet.

transmission. This seems somewhat unexpected, but apparently the loss of selection in one plane is compensated by better selection in the other plane.

\section{CONCLUSION}

The purpose of this study has been a comparative assessment of the focusing properties of a solenoid and a quadrupole triplet in the context of laser accelerated protons (or ions). Possible application of this acceleration method can be envisioned in the field of particle therapy; but also in proton radiography in areas, where energies of the range of 50-100 MeV or above, moderate integrated intensities but high peak intensities in time scales of a ns or less are needed. The relatively large initial angular and energy spreads are a challenge for all of these applications. Their immediate consequence-for both solenoid and quadrupolar systems - is that due to chromatic effects the initially small emittances cannot be conserved and fully utilized.

In terms of transmission it is found that "equivalent" systems - same geometrical length and apertures-give only the relatively small reduction in transmission of $25 \%$ for the triplet vs the solenoid. This is owed to the unsymmetric focusing of quadrupoles, although the disadvantage in the defocusing plane of the first quadrupole is partly compensated by an advantageous focusing in the other plane. For increasing energies-practically above a few $\mathrm{MeV}$ - the weaker focusing properties of solenoids require pulsed or superconducting technology, whereas pole-tip fields of a doublet or triplet can remain within room-temperature iron saturation at all energies. This appears to be a clear advantage for quadrupoles especially in future therapy applications, where short-term and fully controlled changes of energy and magnetic rigidity are required.

The large energy spreads lead to a dominance of chromatic effects, which can be used for energy selection. Equivalent solenoid and triplet systems are equally suitable for this purpose in spite of the strongly differing chromatic coefficients in $x$ and $y$. The $25 \%$ transmission reduction for the triplet system is approximately propagated through the energy selection aperture-subject to fine-tuning according to these chromatic coefficients. Chromatic effects also lead to inevitable correlations between energy and transverse position, which cannot be ignored for therapy applications. In Ref. [10] it is shown that properly placed scatter targets can be used to remove these correlations.

The role of space charge and geometric aberrationsdominant for short solenoids-needs further consideration even though they are not expected to alter the major conclusions. Noting that solenoid focusing is independent of the charge, the neutralizing comoving electrons, which are always present in laser acceleration, will be strongly focused towards the axis [28]. In the triplet, instead, these electrons will be defocused to the aperture when entering the first quadrupole, which may have a (probably small) effect on the quality of focusing and needs to be further explored.

[1] R. A. Snavely et al., Phys. Rev. Lett. 85, 2945 (2000).

[2] P. McKenna, Phys. Rev. E 70, 036405 (2004).

[3] B. M. Hegelich, B. J. Albright, J. Cobble, K. Flippo, S. Letzring, M. Paffett, H. Ruhl, J. Schreiber, R. K. Schulze, and J. C. Fernández, Nature (London) 439, 441 (2006).

[4] A. Gaillard et al., Phys. Plasmas 18, 056710 (2011).

[5] S. V. Bulanov and V. S. Koroshkov, Plasma Phys. Rep. 28, 453 (2002).

[6] E. Fourkal, B. Shahine, M. Ding, J. S. Li, T. Tajima, and C. M. Ma, Med. Phys. 29, 2788 (2002).

[7] V. Malka et al., Med. Phys. 31, 1587 (2004).

[8] M. Roth et al., Phys. Rev. Lett. 110, 044802 (2013).

[9] T. Burris-Mog et al., Phys. Rev. ST Accel. Beams 14, 121301 (2011).

[10] I. Hofmann, J. Meyer-ter-Vehn, X. Yan, and H. Al-Omari, Nucl. Instrum. Methods Phys. Res., Sect. A 681, 44 (2012); I. Hofmann, WIPO Patent Application No. WO/ 2012/080118.

[11] C.-M. Ma, I. Veltchev, E. Fourkal, J. S. Li, W. Luo, J. Fan, T. Lin, and A. Pollack, Laser Phys. 16, 639 (2006). 
[12] P. R. Bolton, T. Hori, H. Kiriyama, M. Mori, H. Sakaki, K. Sutherland, M. Suzuki, J. Wu, and A. Yogo, Nucl. Instrum. Methods Phys. Res., Sect. A 620, 71 (2010).

[13] S. D. Kraft et al., New J. Phys. 12, 085003 (2010)

[14] J. Metzges, T.E. Cowan, L. Karsch, S.D. Kraft, J. Pawelke, C. Richter, T. Richter, K. Zeil, and U. Schramm, Nucl. Instrum. Methods Phys. Res., Sect. A 653, 172 (2011).

[15] T. Toncian et al., Science 312, 410 (2006)

[16] F. Nuernberg, A. Friedman, D. P. Grote, K. Harres, B. G. Logan, M. Schollmeier, and M. Roth, J. Phys. Conf. Ser. 244, 022052 (2010).

[17] M. Schollmeier et al., Phys. Rev. Lett. 101, 055004 (2008)

[18] M. Reiser, Theory and Design of Particle Beams (Wiley-VCH, Weinheim, 2008), 2nd ed., p. 102.

[19] http://irfu.cea.fr/Sacm/logiciels/index3.php.
[20] O. Klimo, J. Psikal, J. Limpouch, and V. Tikhonchuk, Phys. Rev. ST Accel. Beams 11, 031301 (2008).

[21] X. Zhang, B. Shen, X. Li, Z.g Jin, F. Wang, and M. Wen, Phys. Plasmas 14, 123108 (2007).

[22] A. Macchi, F. Cattani, T. Liseykina, and F. Cornolti, Phys. Rev. Lett. 94, 165003 (2005).

[23] T. Esirkepov, M. Borghesi, S. Bulanov, G. Mourou, and T. Tajima, Phys. Rev. Lett. 92, 175003 (2004).

[24] A. Henig et al., Phys. Rev. Lett. 103, 045002 (2009).

[25] A. Henig et al., Phys. Rev. Lett. 103, 245003 (2009).

[26] X. Q. Yan, H. Wu, Z. Sheng, J. Chen, and J. Meyerter-Vehn, Phys. Rev. Lett. 103, 135001 (2009).

[27] I. Hofmann, J. Meyer-ter-Vehn, X. Yan, A. Orzhekovskaya, and S. Yaramyshev, Phys. Rev. ST Accel. Beams 14, 031304 (2011).

[28] A. Almomani, M. Droba, U. Ratzinger, and I. Hofmann, Phys. Rev. ST Accel. Beams 15, 051302 (2012). 